\title{
La vinculación social universitaria: un camino hacia la pertinencia social
}

\author{
University social linkage: a path towards social relevance
}

Zaide Patricia Seáñez Martínez $\bowtie$

Universidad Iberoamericana Torreón

zaide.seanez@iberotorreon.edu.mx

Torreón Coahuila, México

\section{Víctor Hugo Guadarrama Atrizco}

Instituto de Estudios Superiores de la Ciudad de

México "Rosario Castellanos"

Ciudad de México, México

$\bowtie$ Autor por correspondencia

Cómo referenciar:

Seañez Martínez, Z. P., \& Guadarrama Atrizco, V. H. (2022).

La vinculación social universitaria: un camino hacia la pertinencia social.

Emerging Trends in Education, (4)8A, 80-93

https://doi.org/10.19136/etie.a4n8A.4720

Disponible en:

https://revistas.ujat.mx/index.php/emerging

DOI:

https://doi.org/10.19136/etie.a4n8A.4720

Recibido: Aceptado: Publicado:

10/09/2021 05/01/2022

\begin{abstract}
Resumen:
Históricamente las universidades han sido elemento clave del desarrollo socioeconómico, político y tecnológico de las naciones. Para ello requieren establecer una clara y efectiva relación con la sociedad, a lo que comúnmente se le ha llamado vinculación. Dada la importancia de este vínculo, el presente artículo tiene como objetivo revisar la evolución del término que permita conocer en qué se ha centrado el análisis, y a través de ello identificar el grado de importancia de la dimensión social en esta relación. Para lograr este cometido se hace una revisión histórico-teórica sobre la concepción de la vinculación universitaria con su entorno. La revisión arroja que hay poco consenso en la forma de llamarla y definirla, pues se corre el riesgo de caer en generalizaciones o en reduccionismos. Se ha privilegiado el estudio de la vinculación universidad - empresa, por lo que este trabajo propone el concepto de vinculación social universitaria a manera de enfoque para examinar cómo las universidades interactúan con otros actores sociales y hacia todos los ámbitos de la realidad. Es relevante destacar que la vinculación es una estrategia para la pertinencia social, pero no se profundiza en esta última, sino que se esboza como una futura línea de investigación.
\end{abstract}

Palabras clave: Vinculación social; responsabilidad social; pertinencia social; dimensión social; actores sociales.

\begin{abstract}
:
Historically, universities have been a key element of socio-economic, political and technological development of nations. This fact has required a clear and effective relationship with society, which has commonly been called as linkage. Given the importance of this link, the purpose of this article is to review the evolution of the term in order to know what the analysis has focused on, so it allows us to identify the degree of social dimension importance in this relationship. To achieve this task, a historical-theoretical review is carried out of the conception of the university linkage with its environment. This review shows that there is little consensus on how to call it and define it, since there is a risk of falling into generalizations or reductionisms. The study of university-company linkage has been privileged, so this paper proposes the concept of university social linkage as an approach to examine how universities interact with other social actors and towards all areas of reality. It is relevant to highlight that university linkage is a strategy for social relevance, which it is not explored in depth in this document, but is outlined as a future line of research.
\end{abstract}

Keywords: Social bonding; social responsability; social relevance; social dimension; actores sociales. 


\section{La vinculación social universitaria: un camino hacia la pertinencia social}

\section{| Introducción}

Ante escenarios complejos como el de la Cuarta Revolución Industrial, los desafíos relacionados con la desigualdad social y el deterioro del medio ambiente, es necesario repensar y revalorar el rol social de la educación superior, pues sus funciones sustantivas deben proyectarse más allá de la docencia, la investigación o la extensión universitaria. Implica serias transformaciones en modelos y sistemas educativos que prioricen la formación de profesionales con nuevas competencias para trabajos que aún no existen. El papel de la educación superior se torna más relevante; se requiere impulsar el continuo análisis de la realidad y la conciencia social en el proceso formativo dentro del marco de la sostenibilidad, pues es preciso asumir corresponsabilidad ciudadana para contribuir al desarrollo de las naciones (Martínez et al. 2021). Como afirma la Organización de las Naciones Unidas para la Educación, la Ciencia y la Cultura (Organización de las Naciones Unidas para la Educación, la Ciencia y la Cultura [UNESCO], 2015), el espacio de aprendizaje debe trascender al aula, invitándonos a crear entornos de enseñanza que propicien la equidad social y la solidaridad mundial.

La universidad ha transformado su manera de ver al mundo y relacionarse con él. Esto exige analizar continuamente la forma en que se relaciona con su entorno para ser socialmente pertinente. Sus propósitos se alcanzan mediante el desempeño de las funciones sustantivas. A la relación que la universidad establece con su entrono se le ha conocido como vinculación; es oportuno ampliar el abordaje desde la perspectiva social, pues las Instituciones de Educación Superior (IES) son agentes que se adecúan a las exigencias globales en función de sus capacidades y las oportunidades que les brinde el entorno.

El objetivo de este texto es que, a partir de los vacíos y debates encontrados en la revisión documental, se proponga un concepto que resalte la dimensión social en el quehacer universitario. Este concepto es el de vinculación social universitaria, que hace referencia al modelo conformado por procesos y prácticas mediante las cuales las instituciones de educación superior interactúan con la estructura social en la dinámica del desarrollo cultural, político, económico, científico y ambiental, con el fin de ser pertinentes. Esta noción destaca la relación de las IES con los sectores sociales vulnerables, con una investigación enfocada en problemas apremiantes o que desarrollan una extensión más solidaria, lo que contribuya a mejorar la calidad de vida de una comunidad. Es un término útil para futuras investigaciones y poder analizar el impacto que la universidad tiene en la sociedad; evaluar qué tan efectiva es para diagnosticar y responder a las necesidades sociales mediante la aplicación de sus recursos y la ejecución de sus funciones sustantivas. Así se muestra que la educación no es únicamente instrumento de progreso económico, sino también un mecanismo para provocar mejores condiciones (Kromydas, 2017), o refutar la idea de que es una institución de la estructura social que impone arbitrariedad cultural (Molina, 2016).

\section{| Método}

Se realizó una revisión documental sobre la evolución de la universidad para identificar cómo ha sido su relación con el entorno. Se repasaron conceptos, posturas y alcances a fin de conocer algunas lagunas o debates sobre este vínculo bajo el enfoque de la perspectiva 
social. Los criterios seguidos en la exploración fueron:

(a) Palabras relacionadas con vinculación universidadsociedad, vinculación universidad-empresa, universidadsector productivo o industrial; (b) Documentos que traten conceptos de responsabilidad social universitaria, pertinencia social y tercera misión, términos asociados con la vinculación; (c) Literatura sobre nuevas nociones relacionadas al rol social de las instituciones de educación superior, tales como universidad emprendedora, universidad para el desarrollo y universidad empresarial, y (d) Literatura de autores de Europa, Estados Unidos, Latinoamérica y México de veinte años a la fecha.

Tabla 1

Tipos de universidades identificadas según la época histórica

\section{| Aspectos Teóricos}

\section{Evolución de la universidad}

Existen diversas formas de clasificar a las universidades a lo largo del tiempo. Para América Latina, Tünnermann (2001) identificó cuatro tipos: la universidad colonial, la republicana, la moderna y la contemporánea, descritas en la Tabla 1.
Tipo

Colonial

Republicana

Moderna

Contemporánea

\section{Características}

Fundada por los españoles en Hispanoamérica tomó como ejemplo la Universidad de Salamanca y la de Alcalá de Henares. No se utilizaba el término universidad, sino "estudio" (general o particular). Universidades católicas, públicas y privadas; pontificias y reales.

El latín fue el idioma universitario.

La Teología como facultad nuclear con la preocupación de salvar al hombre.

Estableció las bases de un autogobierno universitario, mediante claustros, con la participación estudiantil en las decisiones universitarias.

No participó, en general, en los movimientos de Independencia.

Liberales y conservadores pugnaron por el dominio de la universidad, por lo que la República implementó el modelo francés, sin correspondencia con las necesidades de América.

Su misión se concretó en promover la unidad y estabilidad política, alejada de la ciencia y la cultura, al servicio de las clases dominantes y el Estado.

Justo Sierra fundó en 1910 la Universidad Nacional de México, que buscaría representar la expresión de lo mexicano.

Surgida del modelo Humboldt reintroduce la ciencia y la investigación bajo el principio de libertad académica. Lo más importante es la investigación científica y la formación humana.

Impulsada por la Ley Morrill de 1862, mediante la cual el gobierno federal cedió tierras a quienes fundaran colegios, que se convertirían en universidades estatales. Predominaron las universidades privadas. Estrecharon el vínculo con la empresa productiva.

Los principales aportes fueron la introducción del Departamento como unidad académica básica, la asociación entre investigación y docencia, la revalorización de la cultura general y la promoción de la vinculación entre trabajo y estudio.

La Ley de Veteranos de 1945 impulsó fuertemente el nacimiento de sistemas universitarios en Estados Unidos.

\section{Se identifican las tres funciones sustantivas: docencia, investigación, y difusión o extensión.}

Atención e interés por los problemas sociales que surgen en su entorno, por lo que pasa de una educación elitista a una educación de masas, permeada por los avances en la ciencia y la tecnología. Surge el concepto de educación permanente o continua, por lo que abrió sus puertas a toda la población. Buscó poner el conocimiento al alcance de todos y modificó los métodos de enseñanza.

La universidad debía sobresalir como institución social que pone la ciencia y sus recursos al servicio del hombre.

Dos aspectos de gran trascendencia de este tipo de universidad son la función crítica de la sociedad y la función reconstructiva del orden social.

Nota: Tünnermann (2001). 
Otra clasificación es de Malagón (2003) quien resume lo propuesto por varios autores en función de la relación de la universidad con el entorno social (Tabla 2).

\section{Tabla 2}

Otros tipos de universidades según García Garrido

\begin{tabular}{cl}
\hline Tipo & \multicolumn{1}{c}{ Características } \\
\hline Oxbridge & $\begin{array}{l}\text { Modelo inglés que mezcla la Universidad de Cambridge y la de Oxford. Promo- } \\
\text { vió la educación general y liberal en medio del saber universal, correspondiente } \\
\text { al modelo de organización social y económica de libre cambio y abierta. }\end{array}$ \\
\hline Napoleónico & $\begin{array}{l}\text { Amplio dominio en gran parte del mundo. Enseñanza profesional uniforme, } \\
\text { con una organización institucional acorde al modelo económico cerrado y au- } \\
\text { tárquico. }\end{array}$ \\
\hline \multirow{2}{*}{ Humboldtiano } & $\begin{array}{l}\text { Modelo alemán con fuerte relación entre docencia e investigación para la pro- } \\
\text { ducción del conocimiento ligado a la disciplina. Se estructura por departamen- } \\
\text { tos académicos. Hay un interés genuino por la ciencia. }\end{array}$ \\
\hline
\end{tabular}

Nota: Malagón (2003).

Brunner estudió, en 1985, dos modelos de universidad: la elitista, tradicional y autónoma, y la moderna y heterónoma, según su grado de modernidad y su vinculación con la sociedad (Tabla 3).

\section{Tabla 3}

Clasificación de universidades según Brunner

\begin{tabular}{cl}
\hline Tipo & \multicolumn{1}{c}{ Características } \\
\hline Elitista, tradicional y autónoma & $\begin{array}{l}\text { Anclada en las sociedades predominantemente agrarias y de bajo desa- } \\
\text { rrollo industrial. Desligada de los procesos sociales, introyectada en su } \\
\text { ideal y por ende poco pertinente socialmente. }\end{array}$ \\
\hline \multirow{2}{*}{ Moderna y heterónoma } & $\begin{array}{l}\text { Anclada en los procesos de modernización, urbanización y masificación } \\
\text { de la educación. Integrada en las dinámicas sociales y con mayor capaci- } \\
\text { dad de intervención institucional. }\end{array}$ \\
\hline
\end{tabular}

Nota: Malagón (2003).

En el marco de la sociedad de la información y el conocimiento la universidad ha tendido a adaptarse a los distintos cambios socioeconómicos, políticos, geográficos, tecnológicos, ambientales, etc. Algunos de los factores observados (Malagón, 2006) hacen referencia a lo siguiente: (i) La universidad de la sociedad industrial está basada en la cultura empresarial, orientada a dar respuesta a los intereses productivos y del mercado; (ii) El modelo económico neoliberal influye en el sector educativo a través de las políticas de los organismos multinacionales, sacrificando el bienestar social; (iii) Las universidades latinoamericanas no han podido resolver problemas básicos en el tema de educación, y les cuesta más trabajo integrar sus funciones sustantivas a temas de innovación, emprendimiento y desarrollo tecnológico; en consecuencia, tienen un grado bajo de relación con el entorno; (iv) La educación superior se ha masificado, lo que no implica mayor calidad, y (v) Los recursos gubernamentales han disminuido (para las universidades públicas).

Las tendencias en el rol de las universidades generan nuevos términos. Algunos de ellos se resumen en la Tabla 4, extraída de varios autores: 
Tabla 4

Tendencias para la relación universidad - sociedad

Tendencias

Universidad empresarial

Universidad para el desarrollo

\section{Características}

Formar profesionales con las competencias para adaptarse a las condiciones cambiantes. Estructura orientada a promover la innovación y el emprendimiento, a través de incubadoras de negocios. Coordinación entre docencia, investigación y necesidades sociales y productivas de una región (González et al., 2018).

Según Etzkowitz (2004) interactuar con el gobierno, la industria y otros actores son la clave para lograr los objetivos. Combina la enseñanza, la investigación y la tercera función de capitalizar el conocimiento en pro del crecimiento económico.

Röpke (1998) la define como una institución que tiene estructura y organización empresarial, con una comunidad de empresarios universitarios.

Formulación contemporánea para nuevos modelos de universidad, conjuga la "enseñanza, la investigación, la extensión y otras actividades en el medio, para sumar esfuerzos con muy diversos actores sociales en pro del Desarrollo Humano Sustentable" (Arocena y Sutz, 2016, p. 5).

El desafío es promover una enseñanza para contextos diversos y combinar educación y trabajo. Esto implica atender problemas colectivos de los sectores que han quedado marginados, donde los estudiantes se conviertan en agentes de cambio (Arocena y Sutz, 2016).

Nota: Elaboración propia con información de autores citados en la misma tabla.

Como muestra el análisis anterior la universidad va evolucionando y se modifica en función del contexto. Esto implica enfrentarse a nuevos retos y desafíos con impacto directo en el rol que juega en la dinámica social. Tal es el caso de la situación generada por la crisis del COVID-19 en 2020, la cual ha puesto en jaque los modelos formativos en todos los niveles. La universidad se vio obligada a cambiar sus procesos y prácticas. El confinamiento flexibiliza las restricciones de enseñanza, aprendizaje y transferencia de conocimiento gracias a la educación en línea. Esta situación abre nuevas posibilidades para la vinculación universitaria, lo que permite que las IES tengan la posibilidad de incrementar su participación en el contexto internacional, tal como lo explica Ruiz Gutiérrez, citado en Zapata et al. (2021), a lo que se llamado paradiplomacia universitaria.

En este sentido se entiende que la hegemonía en la producción del conocimiento, así como la formación y educación de las élites dirigentes, lo que ubicó a las universidades en un contexto de acciones diplomáticas hacia el exterior en una posición destacada dentro del sistema de instituciones. Por lo tanto, esta participación constante de las universidades las ubica como un nuevo actor en el sistema internacional capaz de ejercer diplomacia paralela a través de ciertas acciones como la internacionalización de la educación entre otras (Zapata et al., 2021, p. 237).

Hay actividades de vinculación que ayudan a la paradiplomacia como la educación continua en modalidad virtual, la internacionalización de la planta docente, la proyección internacional de la investigación y servicios de consultoría, así como la búsqueda de financiamiento externo.

Estos conceptos proyectan nuevas misiones para las instituciones en el futuro. La evolución de su rol dependerá de comprender los retos y desafíos presentes y futuros, sin perder de vista la pertinencia social de su existir y la importancia del vínculo entre quehacer universitario y bienestar social.

\section{Antecedentes del concepto de vinculación}

El rol de las universidades está estrechamente relacionado con los cambios socioeconómicos de las regiones y hay diversidad de motivos por los cuales buscan vincularse 
con su entorno. Existe una relación recíproca entre IES y sociedad, pues se impactan mutuamente. En este apartado se hace una revisión de la literatura de este concepto.

\section{a) Origen y evolución del concepto de vinculación.}

Gould (2009) afirma que la vinculación ha sido parte del ámbito de la educación superior por más de un siglo. Sitúa sus inicios en Estados Unidos, en 1862, cuando el Congreso aprobó el Acta Morrill "Land Grant Colleges”, lo que originó un crecimiento de los colegios para apoyar a los empresarios dedicados al ramo agrícola. Este mismo autor la define como:

...el conjunto comprensivo de procesos y prácticas planeadas, sistematizadas y continuamente evaluadas, donde las funciones sustantivas y administrativo-financieras de una IES se relacionan internamente entre unos y otros, y externamente con personas y organizaciones públicas y privadas, con el propósito de realizar acciones de beneficio mutuo que: (i) Fomenten la investigación, la innovación y el desarrollo de la base científica y tecnológica; (ii) aumenten la competitividad y las ganancias de las empresas colaboradoras; y (iii) promuevan la comunicación, interacción y colaboración entre las diversas personas y organizaciones involucradas en programas y proyectos enlace (pp. 49-50).

Gould (2009) destaca que los recursos, procesos, actividades y servicios de las instituciones, así como la combinación de las funciones sustantivas trabajan en pro del bienestar de la sociedad.

Otros autores han ahondado en el desarrollo de esta tarea en Europa. Blas (2017) aduce que el Reino Unido fue el primer país europeo que impulsó decididamente esta actividad institucional, con los polythecnics; o que en
Alemania aparecieron las Fachhochschulen con el firme propósito de capacitar a los profesionistas en los negocios e industria. En Holanda trabajan fuertemente para que sus alumnos se conviertan en empresarios, mientras que en Finlandia se apoya la relación que desarrolla portafolios de servicios profesionales para el sector productivo. España se ha convertido en un fuerte promotor de la colaboración universidad-sociedad en todas las regiones del país (Gould, 1997).

El concepto ha sido entendido como el mecanismo mediante el cual las universidades buscan extender sus actividades fuera de su entorno académico para entablar una relación con los sectores y actores que son de su particular interés. Con el paso del tiempo se ha incursionado con mayor frecuencia en actividades de investigación para asistir a la industria, gobierno y sociedad. Ahora se integra el tema de sustentabilidad como eje transversal de su quehacer.

La misión universitaria, según Davyt y Cabrera (2014) ha evolucionado en la historia. Señalan que Napoleón imitó los cambios intelectuales del Reino Unido; reorganizó instituciones para formar profesionales que se incorporarían a las funciones del Estado. En Alemania el avance de esta labor fue orientado por los planteamientos de Whilhem Von Humboldt, cuyo modelo requirió la reorganización de las instituciones públicas hacia una formación más completa, que integrara la investigación a la enseñanza (Jaspers, 1959). La vinculación se consolidó a través de la producción de conocimiento específicamente para ámbitos productivos.

Estados Unidos se basó en el modelo alemán, salvo que se diferenció con una clara intención de vínculo con la sociedad con mayor flexibilidad y capacidad de respuesta ante las demandas sociales. A finales del siglo XIX fortalecieron su autonomía y misión social como universidades (Davyt y Cabrera, 2014). 
Martínez et al. (2010) exponen que hay dos vertientes en la historia del concepto de vinculación: que es inherente a la aparición de la universidad o que es un proceso histórico definido por las condiciones del momento. Si la educación ha caminado a la par del desarrollo económico, social y tecnológico de las naciones, enfrentando obstáculos para desarrollar capacidades diversas y adaptarse a los cambios estructurales para lograr su pertinencia social, se coincide con la segunda postura. Afirman que el proceso de transformación social se fundamenta en el recurso organizacional más importante: el conocimiento. Al ser las universidades fuente de generación y difusión del mismo se presenta una gran oportunidad para que busquen ser más pertinentes y competitivas, estrechando su vínculo con la sociedad.

Para Ortega y Gasset (1930) la misión universitaria era clara: la enseñanza de las profesiones y la preparación de los futuros investigadores. Posteriormente, en su obra La rebelión de las masas (Ortega y Gasset, 1937) agrega que deben añadir una tercera tarea: el compromiso con la sociedad.

Bueno y Casani (2007) exponen tres enfoques para analizar la tercera misión como estrategia de vinculación: basada en la transferencia de conocimiento, basada en la extensión de las actividades universitarias hacia el desarrollo económico y social, y basada en la función del emprendimiento. Este enfoque fue desarrollado por Clark (1998).

Las modalidades que ha adquirido el término recientemente son variadas. Etzkowitz, Gibbons, Sheen, Clark, Ortega y Gasset son precursores del concepto Tercera Misión (Bueno y Casani, 2007). Sin embargo, quedan dudas sobre la importancia de la dimensión social, pues las contribuciones aún priorizan la relación que existe entre la universidad y la industria, empresa o sector productivo.
García Guadilla (como se cita en Malagón, 2006) establece claramente que la vinculación universitaria debe ser un concepto amplio y social, y aclara:

Sin embargo, creemos que la función social de la universidad no se cumple plenamente, ni a través de la 'extensión' ni a través de relaciones más estrechas con el sector productivo. En el primer caso, porque la idea de extensión, tal y como se ha entendido en el modelo anterior, significa algo que se 'añade' pero que no es sustancial de la universidad. en el segundo caso, porque las relaciones universidad-sociedad en nuestros países deben ir más allá de las relaciones universidad-sector productivo (Malagón, 2006, p. 88).

En 1996 se realizó en La Habana la Conferencia Regional sobre Políticas y Estrategias para la Transformación de la Educación Superior en América Latina y el Caribe, donde se concluyó que hay una "clara necesidad de que la educación superior opere dentro de la sociedad contemporánea con una visión que tome en cuenta el logro de mayor pertinencia, calidad y cooperación internacional" (Alcántar y Arcos, 2004, p. 5).

En el presente siglo se ha introducido el término Responsabilidad Social Universitaria (RSU). Vallaeys y Álvarez (2019) revisaron cómo se concibe en Latinoamérica. Encontraron que ésta redefine la tradicional extensión y proyección solidaria de la universidad al agregar un nuevo enfoque global que considere los impactos administrativos y académicos de los procesos universitarios. Jiménez et al. (2002) la definen como "la capacidad que tiene la universidad de difundir y poner en práctica un conjunto de principios y valores generales y específicos, por medio de cuatro procesos claves: Gestión, docencia, investigación y extensión" (p. 5).

La Asociación de Universidades Confiadas a la Compañía de Jesús en América Latina [AUSJAL] (USJAL, 2009) concibe 
la RSU como: "La habilidad y efectividad de la universidad para responder a las necesidades de transformación de la sociedad donde está inmersa, mediante el ejercicio de sus funciones sustantivas: docencia, investigación, extensión y gestión interna" (p. 15). La perspectiva social exige ser protagonista en los procesos sociales, económicos y políticos, con una capacidad crítica y de cuestionamiento del status quo, con diálogo abierto con el entorno y consigo misma.

Ortega y Gasset (como se cita en Bueno y Casani, 2007), afirma que las universidades deben incorporar a su misión un tercer componente que tiene que ver con "el compromiso con la sociedad y con su tiempo, por lo que ha de depurar un tipo de talento para saber aplicar la ciencia y estar a la altura de los tiempos" (p. 45). A esto le llama Tercera Misión. Bueno y Casani (2007) declaran la relevancia de la innovación y el emprendimiento, compromisos de la universidad como agente de creación y transferencia de conocimiento.

Esta breve revisión del término justifica revisar continuamente el concepto para aproximarse a su comprensión.

\section{b) La vinculación universitaria en México.}

Desde 1920, con Justo Sierra se intentó vincular la universidad con la problemática social; se asume que "la docencia y la investigación habrían de extenderse fuera de las instituciones" (Martínez et al., 2010, p. 2). El papel de las universidades adquirió mayor compromiso con el desarrollo de México. Castañeda (1996) define vinculación como:

Un proceso permanente de interrelación entre las actividades académicas y el quehacer de la sociedad en su conjunto, proceso que debe aportar no sólo conocimientos, respuestas y soluciones a problemas y necesidades presentes y futuras de la sociedad, sino también orientar, retroalimentar y enriquecer los programas de formación de recursos humanos, de investigación científica y desarrollo tecnológico, y de extensión y difusión de la cultura (p. 23).

Esta tarea se torna una cuestión estratégica para el país, como lo cita la Secretaría de Educación Pública (SEP) "crear y fortalecer las instancias institucionales y los mecanismos para articular, de manera coherente, la oferta educativa, las vocaciones y el desarrollo integral de los estudiantes, la demanda laboral y los imperativos del desarrollo regional y nacional" (Secretaría de Educación Pública [SEP]/ Centro de Investigación y Docencia Economícas [CIDE], 2010, p. 5).

Otros autores han abordado el tema: (i) Giacomo Gould Bei es fuerte promotor de la Red Nacional de Vinculación; (ii) Rebeca de Gortari promueve la formulación de políticas para impulsar el vínculo universidad-sector productivo con mayor responsabilidad económica para las instituciones; (iii) Carlos Payán afirma que sólo existe con una sólida política de investigación, con aplicación y desarrollo; y (iv) Leonel Corona e investigadores de la Universidad Nacional Autónoma de México (UNAM) sostienen que es una nueva función de la universidad moderna, producto de la convergencia entre investigación científica y desarrollo tecnológico (Campos y Sánchez, 2005).

Campos y Sánchez (2005) exponen que en México no hay un claro significado sobre el término. Argumentan que no es considerada una tarea en la estructura organizacional, lo que afecta el valor otorgado por la comunidad educativa. También identifican tres visiones o perspectivas del término: la economicista, en la cual la vinculación es una fuente de recursos económicos; la productivista, como proceso de transferencia de tecnología para atender las necesidades del sector productivo-industrial; y la 
fisicalista, según la cual casi cualquier cosa es considerada vinculación. En México se encuentran esas tres perspectivas.

En 1937 la UNAM integró brigadas multidisciplinarias como estrategia para acercar a sus estudiantes a las problemáticas reales, con el fin de que esto contribuyera en su preparación profesional. De este modo, "el servicio social fue definido como la responsabilidad institucional de las universidades para con la sociedad que las sustenta" (Cordera, 1994, p. 3).

\section{c) Mecanismos de medición de la vinculación.}

Hay propuestas sobre cómo medir el grado de impacto de la relación universidad-sociedad, pues al ser un concepto de naturaleza multidimensional ha sido difícil consensuar el alcance de esta actividad. Algunas formas se presentan a continuación.

La Science and Technology Policy Research Unit (SPRU) propone indicadores para medir las actividades de la Tercera Misión (Tabla 5).

Tabla 5

Indicadores de la Tercera Misión propuestos por SPRU

\begin{tabular}{l}
\hline Comercialización de la tecnología \\
\hline Actividades emprendedoras \\
\hline Actividades de asesoría \\
\hline Comercialización y uso de equipamientos universitarios \\
\hline Contratos de investigación con clientes no académicos \\
\hline Colaboración no académica en investigación académica \\
\hline Movilidad de personal académico, científico y técnico \\
\hline Trabajo en prácticas para estudiantes \\
\hline Adecuación activa de la docencia a las necesidades económicas y sociales \\
\hline Actividades de aprendizaje \\
\hline Relaciones sociales \\
\hline Difusión/divulgación no Académica
\end{tabular}

Nota: Molas-Gallart et al. (como se citan en Bueno y Casani, 2007).

Estébanez y Korsunsky (2003) analizan la vinculación desde la actividad de transferencia de conocimiento y definen dos categorías de indicadores de medición (Tabla 6):

Tabla 6

Síntesis de propuesta metodológica de indicadores de transferencia de conocimiento

\begin{tabular}{|c|c|c|c|}
\hline Tipos & Indicador & Modo de cálculo & Niveles de análisis \\
\hline \multirow{2}{*}{$\begin{array}{l}\text { Indicadores } \\
\text { de activi- } \\
\text { dad }\end{array}$} & Indicador de actividad total & $\begin{array}{l}\text { Unidades de análisis (con transferencia) / Unidades } \\
\text { de análisis (totales) }\end{array}$ & \multirow{6}{*}{$\begin{array}{ll}\text { Ámbito institucional } \\
0 & \text { Grupos de I+D } \\
0 & \text { Centros o institutos de I+D } \\
0 & \text { Instituciones científicas } \\
\text { o } & \text { Sector institucional }\end{array}$} \\
\hline & $\begin{array}{l}\text { Indicador de nivel de activi- } \\
\text { dad de transferencia }\end{array}$ & $\begin{array}{l}\text { Cantidad de transferencias / Unidades de análisis } \\
\text { (sólo con transferencias) }\end{array}$ & \\
\hline \multirow{4}{*}{$\begin{array}{l}\text { Indicadores } \\
\text { de orienta- } \\
\text { ción }\end{array}$} & $\begin{array}{l}\text { Indicador del tipo de activi- } \\
\text { dad de transferencias }\end{array}$ & $\begin{array}{l}\text { Cantidad de transferencias según tipo de activida- } \\
\text { des de las transferencias / Cantidad total de trans- } \\
\text { ferencias x } 100\end{array}$ & \\
\hline & $\begin{array}{l}\text { Indicador de orientación } \\
\text { social de las transferencias }\end{array}$ & $\begin{array}{l}\text { Cantidad de transferencias según tipo de desti- } \\
\text { natario de las transferencias / Cantidad total de } \\
\text { transferencias x } 100\end{array}$ & \\
\hline & $\begin{array}{l}\text { Indicador de alcance terri- } \\
\text { torial }\end{array}$ & $\begin{array}{l}\text { Cantidad de transferencias según nivel de alcance } \\
\text { territorial / Cantidad total de transferencias x } 100\end{array}$ & \\
\hline & Indicador de modo & $\begin{array}{l}\text { Cantidad de transferencias según tipo de actividad } \\
\text { y destinatario de las transferencias / Cantidad total } \\
\text { de transferencias x } 100\end{array}$ & \\
\hline
\end{tabular}

Nota: Estébanez y Korsunsky (2003). 
El Manual Iberoamericano de Indicadores de Vinculación de la Universidad con el Entorno Socioeconómico (Red de Indicadores de Ciencia y Tecnología y Observatorio de Ciencia, Tecnología y Sociedad de la Organización de Estados Iberoamericanos [RICYT y OCTS-OEI], 2007) es una propuesta que ha sido utilizada en varias regiones, conocido como el Manual de Valencia. Parte de que las IES son actores claves en el tejido social por la docencia, la investigación y la extensión, que les ayudan a cumplir la misión al vincularse con el entorno. Textualmente, el Manual (p. 11) define como actividades de vinculación: de capacidades en colaboración con agentes no académicos y la elaboración de marcos legales y culturales que orienten la apertura de las universidades hacia su entorno;

b) el uso, aplicación y explotación del conocimiento y de otras capacidades existentes en la universidad fuera del entorno académico, así como la capacitación, la venta de servicios, el asesoramiento y la consultoría, realizados por las universidades en su entorno.

El Manual define tres conjuntos de indicadores (Tabla 7):

Tabla 7

Categorías de indicadores del Manual de Valencia

\begin{tabular}{ll}
\hline \multicolumn{1}{c}{ Categoría } & \multicolumn{1}{c}{ Indicadores } \\
\hline & Estructura organizacional \\
Caracterización institucional & Trayectoria histórica \\
& Población universitaria \\
& Recursos financieros \\
& Dimensión y orientación de la I+D \\
\hline & Desarrollo institucional \\
Capacidades para las actividades de & Producción científica \\
vinculación & Propiedad intelectual \\
& Comercialización de infraestructura física \\
& Emprendimiento \\
\hline & I+D contratada con entidades no académicas \\
& I+D en colaboración con entidades no académicas \\
& Asesoramiento y consultoría \\
& Prácticas en entidades no académicas \\
Actividades de vinculación & Cursos y actividades de formación \\
& Alineamiento curricular \\
& Actividades de extensión \\
& Cooperación al desarrollo \\
& Difusión no académica \\
& Participación en redes \\
\hline
\end{tabular}

Nota: Manual de Valencia (RICYT y OCTS-OEI, 2007).

La Secretaría de Educación Pública y el Centro de Investigación y Docencia Económicas (CIDE) estudiaron la Encuesta Nacional de Vinculación en Instituciones de Educación Superior- sector productivo (Encuesta Nacional de Vinculación en Instituciones de Educación Superior [ENAVI], 2010) para recabar información objetiva sobre el diagnóstico de las capacidades universitarias para vincularse, y así diseñar estrategias para estrechar la relación universidad-empresa.

\section{d) La pertinencia social de la universidad.}

La pertinencia social es entendida en este texto como resultado de la interacción de las universidades con su entorno. Malagón (2003) establece que es parte de la relación universidad-sector productivo. Argumenta que va más allá de su perspectiva economicista de convertirse en una empresa de conocimiento sujeta a las leyes del mercado. "La educación superior debería apuntar a crear una nueva sociedad no violenta y de la que esté excluida 
la explotación" (p. 122).

Espinosa (2018) propone la pertinencia económica, tecnológica y global como elementos de la pertinencia social integral. La económica se refiere al grado en que la formación profesional ayuda a la integración efectiva al mercado laboral. La tecnológica se logra cuando los egresados son formados para hacer uso creativo, crítico, ético y responsable de las tecnologías de información en la resolución de problemas y en la construcción de una sociedad del conocimiento. La global es cuando la educación superior se adapta a los escenarios cambiantes, promoviendo la multi e interculturalidad, así como una mayor inclusión de los grupos minoritarios al proceso formativo.

Espinosa (2018) opina sobre la pertinencia social integral lo siguiente:

Al margen de los discursos cotidianos, creemos que las instituciones educativas tendrían que mantener como tarea propia el abordaje de varios aspectos de la pertinencia. Éstos son complementarios entre sí y se relacionan directamente con el desarrollo de las funciones propias de las universidades públicas: los aspectos global, económico y tecnológico, pero también social, político, cultural, ético, de sustentabilidad, de articulación con el resto del sistema educativo, así como de diseño institucional (p. 116).

Este autor agrega dos pertinencias más: la ética y la sustentabilidad. La primera entendida como la capacidad para articularse con la política pública en pro del desarrollo económico y social, y la de priorizar lo mejor para la comunidad. La segunda se logra al formar ciudadanos que identifican, valoran y respetan los rasgos de su propio contexto, con la conciencia antropológica y ecológica en su formación (Espinosa, 2018).
En resumen, la pertinencia universitaria depende de la habilidad para anticiparse a las necesidades del futuro y de la capacidad para formar a un mayor número de profesionistas aptos para el mercado laboral.

\section{| Aportaciones}

La revisión bibliográfica evidencia la necesidad de ampliar y profundizar la comprensión sobre la vinculación universitaria desde la dimensión social. Esto podrá ser útil para orientar futuros estudios que indaguen cómo puede ser la estrategia que asegure a las instituciones de educación llegar a ser socialmente pertinentes. Los hallazgos encontrados se resumen en los siguientes puntos:

1. Las universidades son consideradas agentes estratégicos en el desarrollo local, nacional e internacional. Su acción e influencia en el entorno se ha ampliado a través de la investigación, de la difusión de la cultura, de la formación continua del recurso humano, del desarrollo tecnológico y de la innovación. La revisión documental permitió detectar nuevos beneficios del quehacer universitario que han impactado aspectos más allá de la función de docencia. Nuevas tareas han aparecido en la agenda universitaria como la formación en la ciudadanía y el cuidado de la Casa Común; se impulsa el emprendimiento y la innovación de manera contundente; aparecen nuevas preocupaciones como la responsabilidad social empresarial, la igualdad de género y el respeto por los derechos humanos, como condiciones necesarias de justicia social. La tarea universitaria debe anticiparse a los desafíos del futuro, tal como afirma Edgar Morin: "la humanidad se encuentra en un momento de incertidumbre en el que se está gestando un futuro que no somos capaces aún de 
ver ni en sus contenidos ni en sus alcances" (como se cita en López, 2021).

2. Se propone que la vinculación sea un constructo concebido dentro de un contexto internacional, nacional y regional, con políticas y estrategias adaptadas a las necesidades de cada entorno, congruente con la misión y propósitos, fincada en las capacidades y motivaciones institucionales. La disciplina más importante en la universidad es la realidad, sus actores y lo que en ella sucede. Esta perspectiva permite que la función de vinculación priorice la pertinencia social, pues involucra y amplía la mirada e impacto hacia todos los ámbitos del entorno.

3. Se encontró la preeminencia de documentos sobre la vinculación de la universidad pública, restando valor a la tarea que desempeña la institución privada. Esto abre la oportunidad de examinar y comparar modelos de vinculación distintos que seguramente aportarán nuevas contribuciones al estudio del fenómeno que ayuden a dar una mayor intención al aspecto social. Es urgente la tarea universitaria de apoyar en la construcción de sociedades auténticamente democráticas.

4. En México son más frecuentes las investigaciones sobre vinculación universidad-empresa, a pesar de que en los documentos rectores de las IES se plasme el interés por la transformación social; de aquí que se fundamente la necesidad de explicitar aún más la perspectiva social al estrechar la relación con otros sectores y actores externos.

5. El impacto que ejercen las IES sobre el entorno no siempre es intencionado o evaluado; se requiere una estrategia institucional lo suficientemente clara para su mejor comprensión e impulso de acuerdo a las características de cada institución y región. Es necesario profundizar en el tema de indicadores de medición en función de la pertinencia social universitaria.

6. Las universidades han evolucionado a la luz de los cambios culturales, políticos y económicos en las diferentes regiones del mundo; por ello es necesaria la transformación permanente y pertinente de la relación que la institución establezca con su medio. Implica que cada comunidad educativa acuerde cómo concibe, implementa y evalúa su relación con la sociedad.

7. Estos hallazgos justifican la importancia de la dimensión social en los modelos para relacionarse con otros actores, y de este modo se prevé como estrategia para profundizar en el logro de pertinencia social. La principal aportación de este trabajo radica en el concepto de vinculación social universitaria.

\section{| Conclusiones}

La universidad siempre ha jugado un papel preponderante en el desarrollo humano, económico y social. Ha demostrado su importancia a través de la formación de talento, de la generación de innovaciones y, lo más importante, a través de la promoción de conciencia social para el desarrollo sostenible. Este trabajo evidencia la necesidad de destacar la relación de las universidades con la sociedad, por ello el concepto de vinculación social universitaria se vuelve un importante aporte. Invita a reflexionar sobre el trascendental papel que juegan las instituciones educativas en los procesos sociales; la universidad se debe a la realidad social, a sus actores y 
lo que en la sociedad acontece. Para resaltar el aspecto social dentro de la vinculación, se revisa la evolución de los modelos universitarios a lo largo del tiempo y la evolución de la concepción misma de la universidad. El análisis de las tendencias de las universidades indica que seguirán siendo relevantes para la sociedad.

En la actualidad se debe avanzar hacia modelos universitarios que pongan en el centro de actuación el tema de la sostenibilidad. Por tanto, la universidad debe estar atenta a la comprensión y análisis de los fenómenos, actuar para proponer soluciones y contribuir a la conformación de sociedades más resilientes y cohesionadas.

Esta investigación destaca también la necesidad de definir y elaborar indicadores que midan el grado y la calidad del vínculo universidad-sociedad, mismos que resalten el desempeño alcanzado. Asimismo, expone las ventajas de elaborar nuevas investigaciones que profundicen en el tema de la vinculación social universitaria como estrategia de la pertinencia social institucional, pues se considera éste el fin último del quehacer universitario.

Se concluye que el vínculo universidad-sociedad debe ser tan pertinente en tanto promueva la formación de personas con las competencias profesionales para el mundo laboral, además de las competencias humanas para la transformación social. Implica una constante adaptación de los modelos de vinculación a los cambios vertiginosos en los modos de producir, consumir o convivir. Las universidades no pueden perder de vista su compromiso de formar ciudadanos para el mundo, respetuosos del medio ambiente y de la diversidad de ideologías. En resumen, la vinculación social universitaria es una buena estrategia para el logro de la pertinencia social.

\section{| Referencias}

Alcántar, V., y Arcos, J. (2004). La vinculación como instrumento de imagen y posicionamiento de las instituciones de educación superior. Revista Electrónica de Investigación Educativa, 6(1), 1-12.

Arocena, R., y Sutz, J. (2016). Universidades para el desarrollo. Editorial UNESCO.

Asociación de Universidades Confiadas a la Compañía de Jesús en América Latina [AUSJAL]. (2009). Políticas y sistemas de autoevaluación y gestión de la responsabilidad social universitaria en AUSJAL. Universidad Católica de Córdova.

Blas, N. (2017). Las universidades y la misión de la vinculación en el Reino Unido: Un marco de referencia para pensar la propuesta de indicadores del Manual de Valencia. Revista Iberoamericana de Ciencia, Tecnología y Sociedad., 12(34), 95-110.

Bueno, E., y Casani, F. (2007). La Tercera Misión de la universidad: enfoques e indicadores básicos para su evaluación. Economía Industrial, 43-59.

Campos, G., y Sánchez, G. (2005). La vinculación universitaria: Ese oscuro objeto del deseo. Revista Electrónica de Investigación Educativa, 7(2), 1-13.

Castañeda, M. (1996). Panorama de la vinculación en México. En M. Sánchez, J. Claffey, y M. Castañeda (Eds), Vinculación entre los sectores académico y productivo en México y Estados Unidos (pp. 21-26). ANUIES.

Clark, B. (1998). Creating Entrepreneurial Universities: Organisational Pathways of Transformations. International Association of Universities and Elsevier Science.

Cordera, R. (1994). Las instituciones de educación superior y el servicio social. Revista de la Educación Superior(90), 1-5.

Davyt, A., y Cabrera, C. (2014). Vinculación Universidad Sociedad y formaciones universitarias: una perspectiva histórica y una tesis actual. Congreso Iberoamericano de Ciencia, Tecnología, Innovación y Educación. OEI. Espinosa, M. (2018). Tendencias recientes de la vinculación universitaria con el entorno. Desafíos relacionados con una pertinencia social integral. Revista Iberoamericana de Educación Superior, 9(26), 110-129. 
Estébanez, E., y Korsynsky, L. (2003). Medición de actividades de vinculación y transferencia de conocimientos científicos y tecnológicos. Red de Indicadores de Ciencia y Tecnología - Iberoamericana e Interamericana, 83-92.

Etzkowitz, H. (2004). The evolution of the entrepreneurial university. International Journal of Techonology y Globalisation, 1(64), 64-77.

González, G., Ferreira, E., y Santos, D. (2018). Desafíos de la universidad emprendedora, acercamientos para su gestión. Revista de Gestao do Unilasalle, 7(1), 69-84.

Gould, G. (1997). Vinculación universidad - sector productivo. Una reflexión sobre la planeación y operación de programas de vinculación. ANUIES/Universidad Autónoma de Baja California.

Gould, G. (2009). La Red Nacional de Vinculación: un avance estratégico para el desarrollo de la vinculación en México. Revista Digital de la Red Nacional de Vinculación, 1.

Jaspers, K. (1959). La idea de la universidad. Sudamericana.

Jiménez, M. (2002). Obervando la Responsabilidad Social Universitaria. Memorias del seminario La Universidad Construye País, la responsabilidad social de la unvierdsidad de cara al Chile de 2010 (pp. 3-19). Proyecto Universidad Construye País.

Kromydas, T. (2017). Rethinking higher education and its relationship with social inequalities: past knowledge, present state and future potential. Palgrave Communications, 3(1), 1-12.

López, M. (2021). Universidades para un cambio de vía. Revista Latinoamericana de Estudios Educativos, 51(3), 305-314.

Malagón, L. (2003). La Pertinencia en la Educación Superior. Elementos para su comprensión. Revista de la Educación Superior, 32(3), 113-134.

Malagón, L. (2006). La vinculación Universidad-Sociedad desde una perspectiva social. Educación y Educadores, 9(2), 79-93.

Martínez, J., Tobón, S., y Soto, J. (2021). Ejes claves del modelo educativo socioformativo para la formación univesitaria en el marco de la transformación hacia el desarrollo social sostenible. Formación univesitaria, 14(1), 53-66.
Martínez, L., Leyva, M., y Barraza, A. (2010). La importancia de la vinculación en las instituciones de educación superior. IMEF.

Molina, M. (2016). La sociología del sistema de enseñanza de Bourdieu: Reflexiones desde América Latina. ARTIGOS, 46(162), 942-964.

Ortega y Gasset, J. (1930). Misión de la Universidad. Revista de Occidente - El Arquero, 1-22.

Ortega y Gasset, J. (1937). La rebelión de las masas. Espasa Calpe.

Red de Indicadores de Ciencia y Tecnología y Observatorio de Ciencia, Tecnología y Sociedad de la Organización de Estados Iberoamericanos [RICYT y OCTS-OEI]. (2007). Manual Iberoamericano de Indicadores de Vinculación de la Universidad con el Entorno Socioeconómico. Manual de Valencia. OEI.

Röpke, J. (1998). The entrepreneurial University: innovation, academic knowledge creation and regional development in a globalized economy. Working Paper of Department of Economics, 15. University of Marburg.

Secretaría de Educación Pública/Centro de Investigación y Docencia Económica [SEP / CIDE]. (2010). ENAVI. Encuesta Ncional de Vinculación en Instituciones de Educación Superior. SEP/CIDE.

Tünnermann, C. (2001). Universidad y Sociedad. Balance histórico y perspectivas desde América Latina. Hispamer.

Organización de las Naciones Unidas para la Educación, la Ciencia y la Cultura [UNESCO]. (2015). Replantear la educación ¿Hacia un bien común mundial? UNESCO.

Vallaeys, F., y Álvarez, J. (2019). Hacia una definición latinoamericana de Responsabilidad Social Universitaria. Aproximación a las preferencias conceptuales de los universitarios. Educación XXI, 22(1), 93-116.

Zapata, M., Berlanga, J., y Salazar, H. (2021). La paradiplomacia universitaria, las transfomaciones de las universidades ante el COVID 19. Política, Globalidad y Ciudadanía, 7(14), 233-253. 